\title{
Kaon electro-production on protons at JLab in Hall A
}

\author{
M. Iodice ${ }^{\mathrm{a}}$ \\ For the E98-108 and JLab Hall A Collaborations \\ INFN Sezione di Roma III, I-00146 Rome, Italy \\ Received: 1 November 2002 / \\ Published online: 15 July 2003 - (C) Società Italiana di Fisica / Springer-Verlag 2003
}

\begin{abstract}
The elementary reaction of kaon exclusive electro-production on protons has been studied in a broad kinematical range at Jefferson Lab in Hall A. Data have been taken at different values of the invariant center-of-mass energy $W$ in the range $W=1.8-2.2 \mathrm{GeV}$, for two values of the transferred 4-momentum $Q^{2}=1.9$ and $2.4(\mathrm{GeV} / c)^{2}$. Each kinematics was measured at different electron beam energies so as to separate the longitudinal $(L)$ and transverse $(T)$ contributions to the cross-section. The $L T$ interference term has also been measured for a limited number of kinematics. The preliminary data are compared to results of different models developed in the framework of hadronic field and Regge theories.
\end{abstract}

PACS. 25.30.Rw Nuclear reactions: Electroproduction reactions

\section{Introduction}

The subject of electromagnetic production of strangeness covers an important part of the experimental program of the three experimental Halls at Jefferson Lab. In this paper we report on the experiment E98-108 [1] carried out in Hall A during different periods in 2001 and 2002. The experiment focussed on the investigation of the elementary mechanism of the associated electro-production of kaon-hyperon pairs from a hydrogen target. The exclusive $\mathrm{H}\left(e, e^{\prime} K^{+}\right) \Lambda, \Sigma$ reaction was studied detecting in coincidence the scattered electrons and the produced kaons in the two Hall A High-Resolution Spectrometers (HRS). The kaon arm had been specially outfitted with two aerogel Čerenkov threshold detectors, designed to provide kaon identification in the measured range of momenta, from 1.7 to $2.6 \mathrm{GeV} / c$.

The main goal of the experiment is to take a highprecision data set for the exclusive $\mathrm{H}\left(e, e^{\prime} K^{+}\right) \Lambda$ reaction over a broad kinematical range with $\sigma_{L}, \sigma_{T}$ and $\sigma_{L T}$ response function separation. In combination with the existing world data on kaon photo- and electro-production it will provide a stringent test of theoretical models. Moreover, the kinematics were chosen so as to study the dependence of the longitudinal cross-section $\sigma_{L}$ with the Mandelstam variable $t$. Such a study, when the reaction mechanism is under control, can be used for a measurement of the kaon electromagnetic form factor, by extrapolating $\sigma_{L}$ to the kaon pole at $t=m_{k}^{2}$ located in the unphysical region of positive $t$.

\footnotetext{
a e-mail: mauro.iodice@roma3.infn.it
}

\section{The $\mathrm{p}\left(\mathrm{e}, \mathrm{e}^{\prime} \mathrm{K}^{+}\right) \Lambda, \Sigma$ exclusive electro-production reaction}

The cross-section of the exclusive $p\left(e, e^{\prime} K^{+}\right) \Lambda$ reaction (with a straightforward extension to the $\Sigma$-hyperon production) can be expressed in terms of the $\gamma^{*}+p \rightarrow K^{+}+\Lambda$ binary process cross-section as

$$
\frac{\mathrm{d}^{5} \sigma}{\mathrm{d} E_{e^{\prime}} \mathrm{d} \Omega_{e} \mathrm{~d} \Omega_{k}}=\Gamma \frac{\mathrm{d} \sigma\left(\gamma^{*}, k\right)}{\mathrm{d} \Omega_{k}},
$$

$\Gamma$ being the virtual photon flux.

In turn the virtual-photo-production cross-section can be expressed in terms of 4 response functions:

$\frac{\mathrm{d} \sigma\left(\gamma^{*}, k\right)}{\mathrm{d} \Omega_{k}}=\sigma_{T}+\epsilon \sigma_{L}+\epsilon \sigma_{T T} \cos 2 \Phi+\sqrt{2 \epsilon(\epsilon+1)} \sigma_{L T} \cos \Phi$, where $\epsilon=1 /\left(1+2|\mathbf{q}|^{2} / Q^{2} \tan ^{2} \vartheta_{e} / 2\right)$ is the photon polarization parameter, and $\Phi$ is the "out-of-plane" angle, that is the angle between the leptonic plane (defined by the incoming and outgoing electrons), and the reaction plane (defined by the direction of the 3-momentum transfer and that of the kaon).

The pieces correspond to the cross-section for transverse $\left(\sigma_{T}\right)$, longitudinal $\left(\sigma_{L}\right)$, transverse-transverse interference $\left(\sigma_{T T}\right)$ and longitudinal-transverse interference $\left(\sigma_{L T}\right)$ kaon production by virtual photons and they only depend on the variables $Q^{2}, W$ (or $s$ ) and $t$.

For "in-plane" measurements, in "parallel" kinematics (3-momentum transfer parallel to kaon momentum), the interference terms vanish, allowing the separation of the longitudinal and transverse parts with a Rosenbluth plot. The $\sigma_{L T}$ interference term can be measured through the "left-right" asymmetry ( $\Phi=0,180$ degrees). 


\subsection{Theoretical models}

To calculate the cross-section of the process under investigation different approaches can be adopted. In principle it could be calculated within the QCD theory making use of quark hadronization models. At Jefferson Lab energies, however, non-perturbative QCD degrees of freedom have to be taken into account. A more reliable approach, in this case, is based on hadronic field theories (Quantum Hadron Dynamics) and makes use of semiphenomenological diagrammatic models (also referred to as isobaric models) where the explicit degrees of freedom are mesons and baryons and the tree level Feynman diagrams in the $s, t$ and $u$ channels can be calculated (see, e.g., [2]).

Contributions to the cross-section from these diagrams are usually calculated in a semiphenomenological way, trying to fit the unknown parameters to the existing data. One of the main problems encountered in this kind of approach is the determination of the proper set of resonances. Moreover, in the fitting procedure, the values of the coupling constants (like the leading coupling constant $\left.g_{K \Lambda N}\right)$ have to be established too. So far, quite a large variety of models in this framework have been developed (see, e.g., ref. [2] for older models and refs. [3,4] for more recent developments), but, at present, with the available photo-production and electro-production data (see sect. 2.2 for references to presently available data) it is not possible to determine unambiguously a given set of propagators and coupling constants.

Another interesting and promising approach, developed recently both for pion and kaon photo- and electroproduction, is based on the Regge trajectory exchanges [5]. The nice feature of this model is that it is simple and elegant, only depending on a few parameters (the $g_{K \Lambda N}$, $g_{K^{*} \Lambda N}^{V}, g_{K^{*} \Lambda N}^{T}$ coupling constants and the kaon form factor parameter(s)) and it fairly well reproduces the main features of all existing data for the reactions $e p \rightarrow$ $e^{\prime} K^{+} \Lambda$ and $e p \rightarrow e^{\prime} K^{+} \Sigma^{0}$. It also possesses the correct $Q^{2}$-dependence of the $\sigma_{L} / \sigma_{T}$ ratio.

\subsection{Present status and E98-108 kinematics}

Presently available photo-production data mainly come from old experiments carried out in the 1970s at Cornell, CalTech, Bonn, Desy, Orsay, (collected in [6]), and more recently from the SAPHIR Collaboration in Bonn [7], which extended the old low-energy data up to about $E_{\gamma}=2.2 \mathrm{GeV}$, and from the GRAAL experiment in Grenoble. The new and old data show large discrepancies at small kaon center-of-mass angles. This also reflects on the uncertainties different models have in calculating photo-production cross-sections at small angles.

Old electro-production data from the same period (taken at Cornell, DESY and CEA) are more sparse and of limited precision. A collection of them can be found, e.g. in [8]. The first precise electro-production data with longitudinal/transverse separation come from a quite recent experiment E93-018 [9] carried out at JLab in Hall C. The experiment demonstrated that the longitudinal response

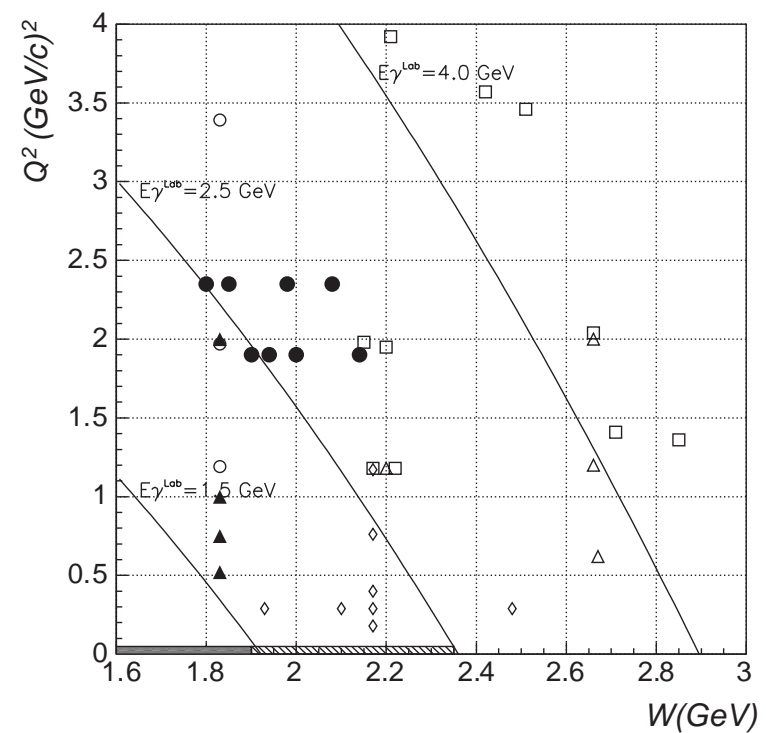

Fig. 1. Kinematics coverage by the world photo-, electroproduction data: open symbols are old data [8] solid triangles refer to [9] and solid circles refer to this experiment. Solid and dashed bars at $Q^{2}=0$ refer to old and new SAPHIR photoproduction data, respectively.

is still large (approximately three-quarters the size of the transverse response) at $Q^{2}=1.8(\mathrm{GeV} / c)^{2}$ and $t=t_{\text {min }}$ or $\theta_{\mathrm{cm}}=0$.

In fig. 1 the $Q^{2}-W$ kinematical plot shows the region covered by the world published data so far, together with the points measured by this experiment (big solid circles). In the figure different regions, in terms of the laboratory photon energy $E_{\gamma}^{\mathrm{Lab}}$, have been identified, where different models have been mostly constrained and are more applicable. For its characteristics, the Regge model is better suited to describe data at high photon energy $\left(E_{\gamma}^{\mathrm{Lab}}>4\right.$ $\mathrm{GeV}$ ), however it has been rather successfully used to describe low-energy electro-production data [9]. Isobaric models, like those from refs. [3] and [4], are more constrained at lower energies $\left(E_{\gamma}^{\mathrm{Lab}}<2.5 \mathrm{GeV}\right)$. As can be seen from the figure, this experiment (E98-108), covers the intermediate region, the main goal being to extend the validity of the different descriptions of the process and, possibly, to find their limits.

\section{The experimental setup}

The experiment took place in Hall A at the Thomas Jefferson National Accelerator Facility's CEBAF accelerator. Electron beams at energies of $3.4,4.2$ and $5.6 \mathrm{GeV}$ and currents in the range of 10-100 $\mu \mathrm{A}$ were incident on liquidhydrogen targets of nominal $4 \mathrm{~cm}$ and $15 \mathrm{~cm}$ lengths. Electrons and kaons were detected in coincidence in the two magnetically symmetric high-resolution spectrometers (HRS). The standard HRS detector packages are described elsewhere [10]. In the case of the hadron arm the standard package has been augmented with two aerogel Čerenkov 


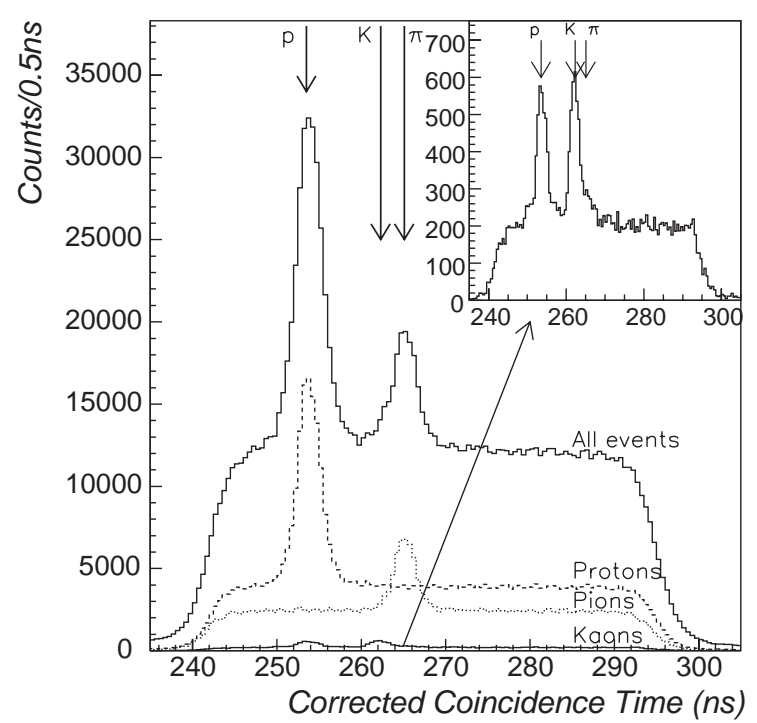

Fig. 2. Time of coincidence spectra with different aerogelcounters-based hadron selections.

threshold counters designed to provide kaon identification in the measured range of momenta, from 1.7 to $2.6 \mathrm{GeV} / c$. The counter with lower refraction index $(n=1.015)$ was used to detect pions above threshold, while the counter with $n=1.055$ detected both pions and kaons. A combination of the information from the two detectors allows the identification of pions, kaons and protons. Figure 2 shows the coincidence time spectrum between the electron and hadron arm. The good quality of hadron identification and kaon selection is qualitatively shown. The exploded part, showing the kaon events, shows clear evidence of the kaon peak and the suppression of the pion one. Still a fraction of protons leak through the selection due to their probability to produce Čerenkov light via electron knock-on processes.

\section{Results}

Figure 3 shows the reconstructed mass of the unobserved baryon in the $\mathrm{H}\left(e, e^{\prime} K^{+}\right)$reaction, in this case either a $\Lambda$ or a $\Sigma$-hyperon. The ratio of these two production crosssections is observed to drop rapidly with $Q^{2}$, however because the phase space acceptance for the two reactions also changes rapidly, final results are dependent on the ongoing acceptance studies. The quality of the particle identification can be judged by the lack of backgroud in the plot.

In the case of the $\Lambda$ production exclusive channel, the unseparated cross-section dependence with $Q^{2}$ has been seen to drop off approximately as $1 /\left(Q^{2}+2.67\right)^{2}$ in agreement with the E93-018 data and also with older experiments [8].

Figure 4 shows the preliminary results on the unseparated cross-sections, as measured for different values of the virtual photon polarization parameter $\epsilon$, for the data at $Q^{2}=2.35(\mathrm{GeV} / c)^{2}, W=1.80,1.85,1.98,2.08 \mathrm{GeV}$, and $t=t_{\min }$ or $\theta_{\mathrm{cm}}=0$. The reported error bars reflect a conservative estimate of what the systematic uncertainties are

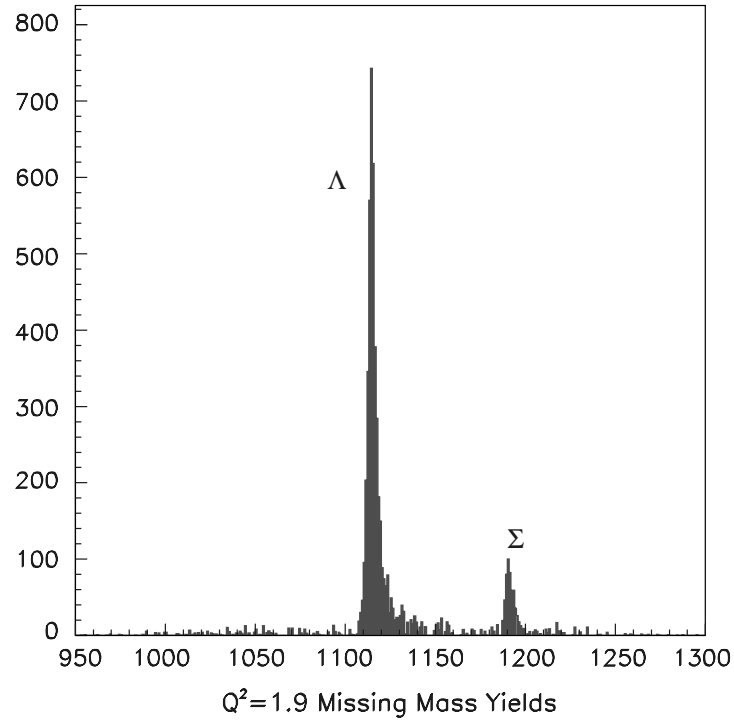

Fig. 3. $\mathrm{H}\left(e, e^{\prime} K^{+}\right)$missing-mass spectrum.

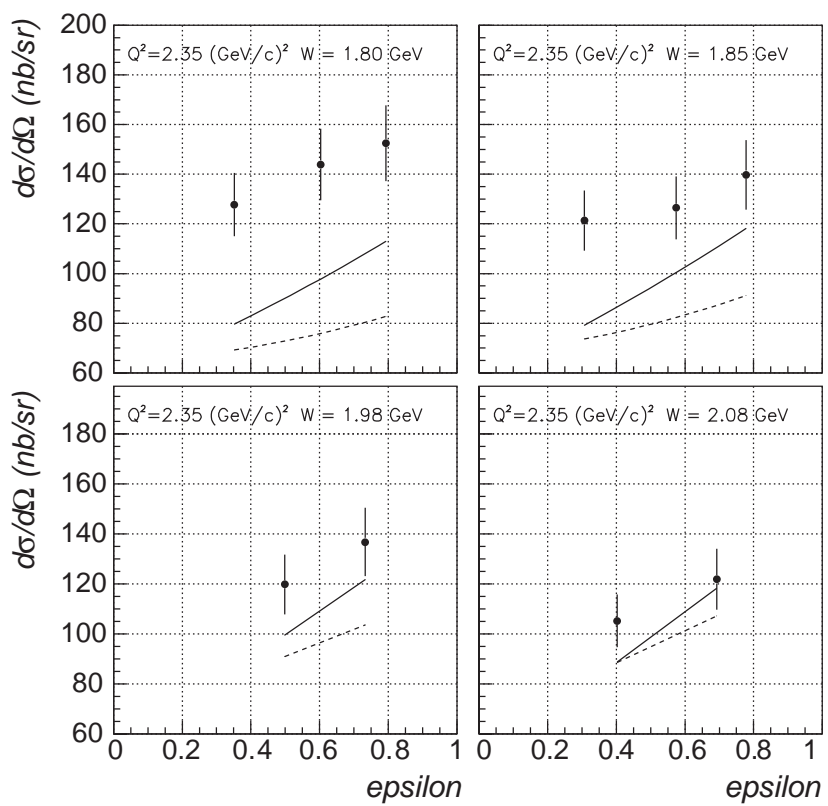

Fig. 4. Preliminary results on the unseparated cross-sections at $Q^{2}=2.35(\mathrm{GeV} / c)^{2}$ and $t=t_{\mathrm{min}}$ or $\theta_{\mathrm{cm}}=0$. The dependence with the photon polarization parameter $\epsilon$ is reported, allowing for $L / T$ response separation. The solid and dashed lines represent the predictions from the Saclay-Lyon [4] and Regge [5] models, respectively.

at this point of the analysis. Due to these uncertainties, an attempt to extract the longitudinal and transverse components of the cross-section from the Rosenbluth plots of fig. 4 has not yet been attempted. The ultimate error bars will be dominated by the statistics, at the level of a few percent. From the plot, however, at the larger momentum transfers of this experiment, as compared to E93-018, the longitudinal cross-section (the slope of the plots in fig. 4) results to be decreasing relative to the transverse, but is still appreciable. 
The figure also shows predictions obtained from the "Saclay-Lyon-A" [4] (solid line) and "Regge" [5] (dashed line) models (recently among the most widely used). Clear discrepancies between the data and the theory exist. However, this is not totally surprising: i) the SL and Regge models are poorly restricted with data (fitting parameters) in the kinematical region where our data lie (high $Q^{2}$, large $W$ ); ii) it is known that $\sigma_{L}$ from the SL model fails to correctly reproduce E3-018 data; iii) the Regge model was mainly developed for high-energy photo-production data, while the electro-production extension was fixed (tuning the form factors) so as to reproduce JLab E93-018 and older data.

\section{Conclusions}

Good-quality data from the first kaon electro-production experiment carried out in Hall A at Jefferson Lab are coming out. The preliminary results (obtained using approximately half of the collected kinematics) if compared with those obtained at lower $Q^{2}$ by the experiment E93-018 [9], show that the longitudinal response decreases faster than the transverse with increasing $Q^{2}$. The adopted theoretical models fail to correctly reproduce the data. As explained in the previous section, this is not surprising, since the parameters of these models had been mainly fixed for kinematical regions quite far from the one measured in the present experiment. Additional results are expected in the coming months.

A close collaboration with theorists is strongly needed to understand the reaction mechanism. The data collected so far, in different kinematical regions, should help in constraining models and, if applicable, in identifying kinematical borders where different descriptions of the reaction have to be adopted. When a satisfactory understanding of the reaction is reached, the good quality of the present experimental data will certainly allow the challenging attempt at the kaon electromagnetic form factor measurement.

The authors would like to acknowledge the collaboration with P. Bydzovsky and M. Sotona. Their theoretical support was kind and useful both for the preparation of the kinematics and during the ongoing phase of the interpretation of the data.

\section{References}

1. P. Markowitz, M. Iodice, S. Frullani, C.C. Chang, O.K. Baker, Jefferson Lab experiment E98-108 (1998).

2. J. Adam, J. Mares, O. Richter, M. Sotona, S. Frullani, Czech. J. Phys. 42, 1167 (1992); M. Sotona, S. Frullani, Prog. Theor. Phys. Suppl. 117, 151 (1994).

3. R.A. Williams, Chueng-Ryong Ji, C.R. Cotanch, Phys. Rev. C 46, 1617 (1992).

4. J.C. David, C. Fayard, G.H. Lamot, B. Saghai, Phys. Rev. C 53, 2613 (1996).

5. M. Guidal, J.M. Laget, M. Vanderhaeghen, Nucl. Phys. A 627, 645 (1997); Phys. Rev. C 61, 025204 (2000).

6. R.A. Adelseck, B. Saghai, Phys. Rev. C 42, 108 (1990).

7. M.Q. Tran, et al., Phys. Lett. B 445, 20 (1998).

8. C.J. Bebek, et al., Phys. Rev. D 15, 594 (1977).

9. G. Niculescu, et al., Phys. Rev. Lett. 81, 1805 (1998).

10. Hall A Collaboration (B.D. Anderson et al.), to be submitted to Nucl. Intrum. Methods; Hall A web site: http://hallaweb.jlab.org/. 